\title{
Cerebral Blood Flow Correlates of Apathy in Alzheimer Disease
}

Ann H. Craig, MD; Jeffrey L. Cummings, MD; Lynn Fairbanks, PhD; Laurent Itti; Bruce L. Miller, MD; Jenny Li; Ismael Mena, MD

Background: Apathy is a pervasive noncognitive neuropsychiatric disturbance in Alzheimer disease, which causes significant caregiver distress. The neuroanatomical substrate of apathy is not well understood.

Objective: To study the relationship between regional cerebral blood flow and the presence and severity of the personality disturbance, apathy, in individuals with Alzheimer disease.

Dosign: Analysis of the relationship between regional cerebral blood flow as measured by single photon emission computed tomography and severity of apathy as measured by the Neuropsychiatric Inventory using an analysis of variance design. We examined regional cerebral perfusion alterations as measured by xenon ${ }^{133} \mathrm{Xe}$ calibrated technetium Tc $99 \mathrm{~m}$ hexamethyl-propyleneamine-oxime single photon emission computed tomography in relation to the presence and severity of apathy.

Sotting: The neurology clinics of the University of California, Los Angeles, UCLA School of Medicine, and Harbor-UCLA Medical Center.

Participants: Thirty-one community-dwelling patients fulfilling National Institute of Neurological and Communicative Disorders and Stroke-Alzheimer's Disease and Related Disorders Association diagnostic criteria for probable Alzheimer disease who had a single photon computed tomographic scan performed within 3 months of administration of the Neuropsychiatric Inventory.

Resuliss: The presence of apathy was associated with more severe prefrontal and anterior temporal dysfunction. These regional cerebral perfusion relationships with apathy were independent of cognitive decline except in the dorsolateral prefrontal cortex.

Conclusions: These results demonstrate the association of apathetic syndromes with prefrontal and anterior temporal regional brain dysfunction and are consistent with similar findings previously reported in other disorders.

Arch Neurol. 1996;53:1116-1120
From the Departments of Neurology (Drs Craig and Cummings) and Psychiatry and Biobehavioral Sciences (Drs Cummings and Fairbanks), University of California, Los Angeles, UCLA School of Medicine; Behavioral Neuroscience Section, Psychiatry Service, West Los Angeles Veterans Affairs Medical Center

(Dr Cummings); and Departments of Neurology (Dr Miller, Ms Li, and Mr Itti) and Radiology (Dr Mena), Harbor-UCLA Medical Center.

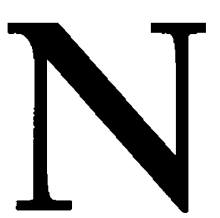

EUROPSYCHIATRIC disturbances are prominent features of Alzheimer disease (AD). Delusions and hallucinations; mood disturbance; changes in personality and demeanor such as apathy, irritability, and disinhibition; and disturbances of sleep, appetite, sexual function, and psychomotor activity occur during the course of the disease. ${ }^{1}$ Personality changes are similar among patients with $\mathrm{AD}$ and are more closely related to the underlying neurobiological alterations of the disease than to premorbid personality characteristics. ${ }^{2}$ Apathy or indifference is the most commonly observed behavioral disturbance in $A D$. Investigators using disparate methods have found prevalances up to $90 \%$. Disengagement with passivity and loss of enthusiasm, interest, empathy, and interpersonal involvement occurs early in the course of $A D$ and is persistent. ${ }^{3-6}$ Apathy has been found to be the most personally distressing behavioral change for caregivers of patients with dementia. ${ }^{7}$ The neuroanatomical substrate of this ubiquitous behavioral disturbance in $\mathrm{AD}$ is not well understood.

Functional imaging studies in AD using positron emission tomography and single photon emission computed tomography (SPECT) have established a characteristic pattern of posterior parietal and temporal association area defects with variable and increasing involvement of premotor and prefrontal regions during disease progression. Asymmetries in functional imaging changes have been correlated with the particular cognitive deficits expected from previous brain lesion studies; disproportionate left hypometabolism is associated with predominant language deficit and more marked visuospatial abnormalities occur in patients with more

\section{See Subjects and Methods on next page}




\section{SUBJECTS AND METHODS}

\section{SUBJECTS}

All subjects were community-dwelling outpatients evaluated at the University of California, Los Angeles (UCLA School of Medicine or Harbor-UCLA Medical Center), neurology clinics between June 1993 and October 1994 for dementia. All patients fulfilling National Institute of Neurological and Communicative Disorders and Stroke- Alzheimer's Disease and Related Disorders Association ${ }^{17}$ diagnostic criteria for probable AD who had a SPECT scan performed within 3 months of administration of the Neuropsychiatric Inventory ${ }^{18}$ (NPI) were included in the analysis. Dementia assessment included the Mini-Mental State Examination (MMSE), ${ }^{19}$ which was used as a measure of dementia severity.

There were 31 patients in the sample. They were predominantly women $(64 \%)$ with an average age of 73.1 years (SD, 8.2 ; range, $54-85$ years). Seven patients $(22 \%)$ were reported to be taking a psychotropic medication at the time of study: antidepressants, 4 ; benzodiazepine, 1 ; neuroleptics, 1 ; and tacrine, 1 . The subgroup receiving psychotropic medications did not differ significantly from those not taking medications as to apathy scores.

\section{SINGLE PHOTON EMISSION COMPUTED TOMOGRAPHY}

All subjects were examined by means of brain-dedicated SPECT that included absolute measurement of cerebral blood flow (CBF) with xenon ${ }^{133} \mathrm{Xe}$ and high-resolution imaging of CBF with technetium Ic $99 \mathrm{~m}$ hexamethyl-propyleneamine-oxime (Tc 99m HMPAO) ${ }^{20} \mathrm{~A} 3$-ring brain-dedicated unit (Headtome II, Shimadzu, Kyoto, Japan) with high-sensitivity collimation was used. Ambient light and noise were minimized during scanning. Cerebral blood flow was measured after inhalation of 1100 MBq of xenon ${ }^{133} \mathrm{Xe}$ gas (DuPont, Billerica, Mass). After ${ }^{133} \mathrm{Xe}$ studies, each subject underwent cerebral perfusion imaging with Tc 99m HMPAO (Ceretec, Amersham, Arlington Heights, Ill). A Tc $99 \mathrm{~m}$ HMPAO (1100-MBq) bolus was injected intravenously via butterfly needle. Scanning began 1 hour after injection after clearance of soft tissue and muscle background activity. Three 16-mm-thick brain slices separated by $32 \mathrm{~mm}$ were acquired simultaneously. Acquisitions were completed in 30 minutes using shifts of $16 \mathrm{~mm}$ to image the interslice space and yielding 12 contiguous transaxial slices. This method achieves spatial resolution of $8 \mathrm{~mm}$ in the cortex and $9.6 \mathrm{~mm}$ in the basal ganglia. The ${ }^{133} \mathrm{Xe}$ measures of absolute $\mathrm{CBF}$ were used to calibrate higher-resolution Tc 99m HMPAO scans (milliliters per $100 \mathrm{~g}$ of tissue) using a linearization correction factor based on the Lassen algorithm. ${ }^{20-23}$

\section{COREGISTRATION WITH AVERAGED-AD MAGNETIC RESONANCE IMAGING AND VALIDATION}

An averaged-AD magnetic resonance imaging (MRI) was created using a multimodality coregistration method. ${ }^{24}$ Ten individual MRIs of the brain of patients with probable AD were iteratively combined resulting in the averaged-AD MRI. There were no significant differences in age or MMSE score between the group of patients with probable $\mathrm{AD}$ whose MRI scans were used to make the averaged-AD MRI and members of the study group. Anatomical regions of interest were drawn by one of us (A.H.C.) on the averaged-AD MRI guided by templates of cytoarchitectonic regions developed by Damasio and Damasio. ${ }^{25}$ Each of the AD cohort SPECT scans was then coregistered with the averaged-AD MRI. The ${ }^{133} \mathrm{Xe}$-calibrated Tc $99 \mathrm{~m}$ HMPAO measure of $C B F$ and variances for each region were calculated using pixel-weighted averages for regions drawn on multiple image slices. Eight regions in each hemisphere were analyzed for each patient with $\mathrm{AD}$ (relevant portions of Brodmann areas [BAs]): orbitofrontal (BAs 10, 11, and 47),

Continued on next page severe right hemisphere dysfunction. ${ }^{8,9}$ More recently, specific psychiatric disturbances including delusions and depression have been associated with regional functional alterations as demonstrated by both positron emission tomography and SPECT. ${ }^{10,11}$

We undertook this exploratory analysis of SPECT regional cerebral blood flow ( $\mathrm{rCBF}$ ) changes in a cohort of individuals with probable AD in an effort to better understand the neurobiological correlates of apathy in this disorder. Based on observations of prominent apathy in frontal brain injury ${ }^{12-14}$ and recent insights regarding the relevance of frontal-subcortical circuits to behavior, ${ }^{15.16}$ we hypothesized that apathy would be associated with prefrontal hypoperfusion in $\mathrm{AD}$ and specifically with more focal anterior cingulate dysfunction.

\section{RESULTS}

\section{PATIENT CHARACTERISTICS}

Severity of dementia ranged from mild to severe with a mean MMSE score of 17.6 (SD, 6.9; range, 0-27). Some degree of apathy was present in $81 \%$ of the patients with probable AD.
Apathy severity was the following: none, $6(19 \%)$; mild, 4 (13\%); moderate, $13(42 \%)$; and severe, $8(26 \%)$. The groups with no or only mild apathy were collapsed as there were no scores of 2 , and a score of 1 indicates a degree of apathy only mildly different from the patient's behavior prior to onset of the illness and the apathetic behavior is noted less than once weekly. This was done to have sufficient cases per cell for the ANOVA. Apathy was the only NPI symptom occurring to a moderate to severe degree in more than half of the patients with AD. Apathy was present in all patients with AD with MMSE scores of less than 17.

\section{${ }^{133}$ Xe-CALIBRATED HMPAO SPECT rCBF}

For the entire group of patients with probable $\mathrm{AD}$, the mean $\mathrm{rCBF}$ was maximal in the mesial occipital cortex $(42.7 \mathrm{mg} / 100 \mathrm{~g}$ per minute), followed by the thalamus (38.7 $\mathrm{mg} / 100 \mathrm{~g}$ per minute), the dorsolateral prefrontal ( $36.4 \mathrm{mg} / 100 \mathrm{~g}$ per minute), parietal ( $32.1 \mathrm{mg} / 100 \mathrm{~g}$ per minute), posterior temporal (30.6 mg/l00 g per minute), anterior temporal $(29.8 \mathrm{mg} / 100 \mathrm{~g}$ per minute $)$, anterior cingulate ( $29.8 \mathrm{mg} / 100 \mathrm{~g}$ per minute), and orbitofrontal (29.4 mg/100 g per minute) regions (Table 1). Mean cortical blood flow of 44.9 in a normal elderly popula- 
dorsolateral prefrontal (BAs 9, 10, and 46), anterior cingulate (BA 24), anterior temporal (BAs 20, 21, 22, 28, 36, and 38 ), posterior temporal (BAs 37 and 41 ), parietal association (BAs 7, 39, and 40), occipital visual cortex (BA 17), and thalamus. Anteroposterior (A-P) cortical CBF ratios were defined as the ratio of pixel-weighted averages of rCBF in the 3 prefrontal (lateral orbitofrontal, dorsolateral prefrontal, and anterior cingulate) and anterior temporal regions divided by that in the posterior temporal and parietal regions.

The reliability of the regional perfusion measurement made by the MRI template-SPECT coregistration method was examined by comparing rCBF measurements in a subgroup of 16 patients. A subset of regions of interest (orbitofrontal, anterior cingulate, dorsolateral prefrontal, and anterior temporal) were drawn by one of us (B.L.M.) on the averaged-AD MRI template using the same neuroanatomical atlas. ${ }^{25}$ The coregistration process was repeated with each patient's SPECT scan, and pixel-weighted mean rCBF was recalculated. The subgroup of 16 did not differ significantly from the whole group in age, MMSE score, or NPI apathy score. Mean correlation coefficient for interrater reliability for $\mathrm{rCBF}$ was $r=0.96,\left(R^{2}=0.93\right)$.

\section{NEUROPSYCHIATRIC INVENTORY}

The NPI was administered to each patient's principal caregiver in the absence of the patient as part of the initial evaluation of dementia. The administration and validation of this instrument has been described previously. ${ }^{18}$ Ten behavioral disturbances (representing changes from premorbid behavior) occurring in patients with dementia are assessed and quantified as to frequency and severity. Behavioral domains explored are the following: delusions, hallucinations, dysphoria, anxiety, agitation and aggression, disinhibition, euphoria, irritability and lability, apathy, and aberrant motor activity. Frequency is rated as 1 to 4 using defined criteria, and severity is scored as 1 to 3 with operationalized definitions. The product (frequency $\times$ severity) of these ratings was calculated for each behavioral change present during the previous month and used in the analysis. The presence of apathy and indifference was established by a positive response to screening queries regarding loss of interest in activities, conversation, household chores, or the external world. Subquestions regarding spontaneity, change in activity level, expression of affection and emotion, interest in friends and family members, and enthusiasm regarding usual interests and initiation of new activities were then asked to further explore the presence of apathetic behavior. Frequency and severity questions are linked to the responses to these subquestions. The NPI domain scores were grouped to reduce the effect of outliers, as follows: none; mild, 1 to 2 ; moderate, 3 to 4; and severe, 6 or more. Validation data for scoring apathy are previously published and address interrater and testretest reliability. ${ }^{18}$

\section{DATA ANALYSIS}

Statistical analyses were performed using BMDP Statistical Software. ${ }^{26}$ Intergroup comparisons were made using analysis of variance (ANOVA). Blood flow differences by brain region among groups with varying severity of apathy were first assessed using ANOVA with repeated measures, with apathy severity level as an independent grouping factor and brain region of interest as a repeated measures factor. Univariate ANOVA was then used to explore the relationship between apathy and blood flow in specific brain regions as well as with the A-P CBF ratio. Analyses of variance and covariance were used to control for the effects of MMSE score and dysphoria, which were significantly correlated with NPI apathy scores, on apathy and rCBF relationships. Pearson correlations ( $r$ ) between rCBF and apathy scores were also performed. None and mild apathy groups were combined for the ANOVA, but not for the correlation analyses. tion using the same SPECT scanner and ${ }^{133} \mathrm{Xe}$ technique has previously been reported. ${ }^{14}$

\section{RELATIONSHIP BETWEEN rCBF AND NPI APATHY SCORE}

The association between apathy and $\mathrm{rCBF}$ varied as to brain region. The interaction of apathy severity groups by brain region was found to be significant by ANOVA with repeated measures $(d f, 21,189 ; F=2.12 ; P<.01)$. Univariate ANOVA of mean $\mathrm{CCBF}$ in relation to apathy severity (Tablo 2) revealed significantly decreased rCBF in the anterior cingulate, orbitofrontal, dorsolateral prefrontal, and anterior temporal regions in those with moderate and severe apathy compared with those with no or minimal apathy. The A-P CBF ratio was also significantly decreased in those with apathy. There were no significant differences in $\mathrm{rCBF}$ in parietal association, posterior temporal or occipital primary visual cortex, or thalamic regions among those with and without apathy. The ANOVA was repeated excluding the 7 patients receiving psychotropic medications without any change in the significance of relationships described above.
As the apathy score was significantly correlated with MMSE score $(r,-0.41 ; P=.02)$, ANOVA and analysis of covariance with MMSE score as a covariate $(d f=3)$ was performed. This showed that significant regional differences survived controlling for MMSE score in the anterior temporal $(\mathrm{F}=2.99 ; \mathrm{P}<.05)$ and orbitofrontal cortices $(\mathrm{F}=3.32 ; P<.04)$. Borderline statistical significance was retained for the anterior cingulate $(F=2.87 ; P=.06)$, but not the dorsolateral prefrontal cortex $(\mathrm{F}=1.59 ; P=.21)$. The difference in the A-P ratio between those with and without apathy remained robust $(F=5.99 ; P=.003)$.

Although dysphoria and apathy were modestly correlated $(r=0.36 ; P=.05)$, dysphoria was significantly related neither to $\mathrm{rCBF}$ variation nor to A-P ratio. The ANOVA and analysis of covariance with dysphoria as a covariate $(d f=3)$ showed that significant relationships between apathy and rCBF survived for the anterior temporal $(\mathrm{F}=3.96 ; P=.02)$, orbitofrontal $(F=4.8 ; P=.009)$, and anterior cingulate regions $(\mathrm{F}=5.07 ; P=.007)$, as well as the A-P ratio $(\mathrm{F}=8.23 ; P<.001)$ and resulted in borderline statistical significance for the dorsolateral prefrontal cortex $(\mathrm{F}=2.89 ; P=.05)$. The ANOVA indicated no significant effect of age or sex on apathy and rCBF relationships. 


\section{COMMENT}

The presence of apathy, characterized by indifference, emotional disengagement, social withdrawal, and loss of interest in $80 \%$ of our AD cohort, is consistent with previous reports. ${ }^{23.27}$ Rubin and colleagues ${ }^{5}$ reported decreased initiative in 39 of 44 patients with mild $A D$ and the presence of a "passive" cluster of behavioral changes in two thirds. Apathy is seen throughout the course of $\mathrm{AD}$ and becomes more pervasive as the disease progresses.

We studied regional differences in CBF in patients with $\mathrm{AD}$ with and without apathy. The presence of apathy was associated with more severe prefrontal and anterior temporal dysfunction, as indicated by hypoperfusion.

There are several possible neurobiological determinants of apathy in $\mathrm{AD}$ including histological changes in prefrontal and anterior temporal cortex and cortical cholinergic dysfunction. Dramatic personality changes have been associated with frontal lobe injury and regional correlates of altered behavior are recognized. ${ }^{15}$ Apathy with impaired motivation, marked indifference to circumstance, and variable degrees of akinesia has been most strongly associated with anterior cingulate damage. ${ }^{13}$ The anterior cingulate cortical-subcortical circuit mediates motivated behavior, and profound apathy is seen with dysfunction of member structures of this circuit. Orbitofrontal and entorhinal afferents provide information regarding

\section{Table 1. Regional Blood Flow Using Xenon \\ ${ }^{133}$ Xe-Calibrated Technotium Tc 99m HMPAO*}

\begin{tabular}{lc}
\hline Brain Region & Mean $( \pm$ SD) rCBFT \\
\hline Orbitofrontal & $29.4(6.7)$ \\
Anterior cingulate & $29.8(7.5)$ \\
Dorsolateral prefrontal & $36.4(8.3)$ \\
Anterior temporal & $29.8(6.0)$ \\
Posterior temporal & $30.6(6.0)$ \\
Parietal & $32.1(5.4)$ \\
Occipital & $42.7(7.2)$ \\
Thalamic & $38.7(6.7)$ \\
\hline
\end{tabular}

*HMPAO indicates hexamethyl-propyleneamine-oxime. All values are milligrams per $100 \mathrm{~g}$ per minute.

†Averaged bilateral regional cerebral blood flow (rCBF) values. the emotional relevance of external stimuli to the anterior cingulate circuit from the internal and external environments. ${ }^{15,16}$ The anterior cingulate cortex is involved in $A D$, and these changes may contribute to the prominent loss of motivation observed in patients with $\mathrm{AD}$. Blunted affect, apathy, and petlike compliance have also been observed in Kluver-Bucy syndrome involving lesions of the amygdala and medial basal temporal lobes. ${ }^{28}$ These structures are subject to marked structural and chemical change in $\mathrm{AD}$. Pathological involvement in $\mathrm{AD}$, including neuritic plaques, neurofibrillary tangles, and neuronal and synaptic loss, is most severe in the medial temporal lobes, inferior parietal association cortices, frontal convexities, and nucleus basalis of Meynert. ${ }^{29}$

The nucleus basalis of Meynert sends cholinergic projections diffusely to the neocortex, but also to the mediodorsal, ventroanterior, and reticular thalamic nuclei ${ }^{16}$ Loss of cholinergic projections directly to frontal cortex or impaired facilitation of thalamocortical activation in AD may have a role in the early and common occurrence of apathy.

Correlations between apathy presence and severity and multiple anterior regions suggest that intact neuronal networks rather than single cortical regions are important for preservation of the complex personality traits of drive, initiative, and interest. Its appearance early in $\mathrm{AD}$ may be primarily related to loss of nucleus basalis of Meynert cholinergic input to prefrontal (primarily anterior cingulate) and subcortical (thalamic) structures, and later worsening may be related both to more profound cholinergic denervation and to pathological involvement of network structures (prefrontal and anterior and medial temporal).

The relationship between apathy and disease severity in $\mathrm{AD}$ is complex. With disease progression there is both progressive decline in cognitive function and greater prefrontal involvement. Disease severity accounted for $42 \%$ of the variance in an apathy scale in one study ${ }^{4}$ Forstl and colleagues $^{30}$ examined neuropathological changes in $\mathrm{AD}$ in relationship to behavioral disturbances including apathy. They found that apathy severity (combined with physical disability and communication failure scores) correlated with both dementia severity and higher medial frontal

Table 2. Regional Cerebral Blood Flow and Apathy Score*

\begin{tabular}{|c|c|c|c|c|c|c|}
\hline \multirow[b]{2}{*}{ Brain Region } & \multicolumn{3}{|c|}{ Mean (SEM) } & \multirow[b]{2}{*}{$\mathbf{F}$} & \multirow[b]{2}{*}{$\boldsymbol{P}$} & \multirow[b]{2}{*}{$\boldsymbol{r}$} \\
\hline & No or Mild & Moderate & Severe & & & \\
\hline Anterior temporal & $34.4(2.4)$ & $27.2(0.8) \dagger$ & $28.4(1.6) \neq$ & 5.9 & .008 & $-0.40 t$ \\
\hline Orbitofrontal & $34.9(2.7)$ & $25.9(0.8) \dagger$ & $28.1(1.4) \ddagger$ & 7.4 & .003 & $-0.43 t$ \\
\hline Anterior cingulate & $36.1(2.6)$ & $26.3(1.3) \dagger$ & $27.8(2.1) \ddagger$ & 7.3 & .003 & $-0.45 t$ \\
\hline Dorsolateral prefrontal & $42.1(3.2)$ & $32.8(1.6) \dagger$ & $35.1(2.0)$ & 4.6 & .02 & $-0.36 t$ \\
\hline Parietal & $34.3(2.2)$ & $30.4(1.2)$ & $32.1(1.5)$ & 1.5 & .24 & -0.17 \\
\hline Posterior temporal & $33.1(2.5)$ & $29.0(1.5)$ & $30.3(1.2)$ & 1.4 & .26 & -0.19 \\
\hline Occipital & $43.3(2.7)$ & $41.9(1.8)$ & $38.2(2.5)$ & 1.1 & .35 & -0.18 \\
\hline Thalamic & $42.5(2.5)$ & $36.8(1,4)$ & $36.9(2.0)$ & 2.7 & .08 & $-0.36 \dagger$ \\
\hline Anteroposterior ratio & $1.07(0.03)$ & $0.95(0.02) \dagger$ & $0.94(0.03) \dagger$ & 9.3 & .001 & $-0.54 \ddagger$ \\
\hline
\end{tabular}

* Data reported as regional cerebral blood flow (rCBF) in milligrams per $100 \mathrm{~g}$ per minute. Pearson correlations (r) are for rCBF and apathy scores; $F$ and $\mathrm{P}$ values are from univariate one-way analysis of variance of CBF by apathy severity level. $\mathrm{P}$ values for $\mathrm{t}$ test comparisons vs no or mild apathy.

$\dagger \mathrm{P}<.01$.

$\ddagger \mathrm{P}<.05$. 
(BA 32), parietal (BA 7), and parahippocampal gyrus tangle counts and with neuronal loss in the hippocampus and nucleus basalis of Meynert. In the current study there was also increased apathy with more severe dementia. Regional CBF relationships with apathy were independent of cognitive decline as measured by the MMSE, with the exception of the association between MMSE score and perfusion of the dorsolateral prefrontal cortex. The observation that dorsolateral prefrontal cortex hypoperfusion was most closely related to MMSE score is in keeping with the reported high correlation $(r=0.58)$ between a frontal cluster of neuropsychological tests ${ }^{19}$ mediated by the dorsolateral convexity ${ }^{31}$ and MMSE score. Marked correlation has also been reported between MMSE and dorsolateral frontal neuropathological changes in brains with $\mathrm{AD}{ }^{32,33}$ Neuronal death is closely related to synapse loss in $A D,{ }^{34,35}$ which, in turn, correlates strongly with decline in cognitive function. This relationship is most marked for the dorsolateral frontal region (BA 46). ${ }^{33}$

Although apathy can occur as part of depressive syndromes and was modestly correlated with dysphoria in our patients, no shared rCBF associations between apathy and dysphoria were apparent in the present study. Previously reported associations between paralimbic frontal and temporal abnormalities on functional imaging studies in primary and secondary depression were not seen. ${ }^{36-38}$ However, the number of patients with AD with dysphoria in our study was small $(n=13)$ and none had major depression.

Our study extends previous observations regarding the association of apathy and prefrontal and anterior temporal dysfunction. It must be viewed as preliminary and exploratory because of the small sample size. The findings are consistent with previous reports of regional affiliations of apathetic behavioral syndromes of various causes. Our investigation adds to the growing number of observations indicating relationships between neuropsychiatric syndromes and regional brain dysfunction in patients with $\mathrm{AD}$.

\section{Accepted for publication June 14, 1996.}

This project was supported by the Department of Veterans Affairs, a National Institute on Aging (NIA) Alzheimer's Disease Center, Bethesda, Md, grant (AG10123), and a NIA training grant (AG00172).

Reprints: Jeffrey L. Cummings, MD, Reed Neurological Research Center, UCLA School of Medicine, 710 Westwood Plaza, Los Angeles, CA 90024.

\section{REFERENCES}

1. Cummings $\mathrm{JL}$, Victoroff $\mathrm{J}$. Noncognitive neuropsychiatric syndromes in Alzheimer's disease. Neuropsychiatry Neuropsychol Behav Neurol. 1990;3:140-158.

2. Petry S, Cummings JL, Hill MA, Shapira J. Personality alterations in dementia of the Alzheimer type. Arch Neurol. 1988;45:1187-1190.

3. Bozzola FG, Gorelick PB, Freels S. Personality changes in Alzheimer's disease. Arch Neurol. 1992;49:297-300.

4. Gilley DW, Wilson RS, Bennett DA, Bernard BA, Fox JH. Predictors of behavioral disturbance in Alzheimer's disease. J Gerontol. 1991:46:362-371.

5. Rubin EH, Morris JC, Storandt M, Berg L. Behavioral changes in patients with mild senile dementia of the Alzheimer's type. Psychiatry Res. 1987:21:55-62.

6. Rubin $\mathrm{EH}_{1}$ Morris $\mathrm{J} \mathrm{C}$, Berg $\mathrm{L}$. The progression of personality changes in patients with mild senile dementia of the Alzheimer type. J Am Geriatr Soc. 1987;35: $72 t-725$.
7. Greene JG, Smith R, Gardiner M, Timbury GC. Measuring behavioral disturbance of elderly demented patients in the community and its effects on relatives: a factor analytic study. Age Aging. 1982;11:121-126.

8. Grady CL, Haxby JV, Schlageter NL, Berg G, Rapoport SI. Stability of metabolic and neuropsychological asymmetries in dementia of the Alzheimer type. Neurology. 1986;36:1390-1392

9. Haxby JV, Grady CL, Koss E, et al. Heterogeneous anterior-posterior metabolic patterns in dementia of the Alzheimer type. Neurology. 1988;38:18531863.

10. Starkstein SE, Vazquez S, Petracca G, et al. A SPECT study of delusions in Alzheimer's disease. Neurology. 1994:44:2055-2059.

11. Mayberg HS. Clinical correlates of PET and SPECT identified defects in dementia. J Clin Psychiatry. 1994;55(suppl):12-21.

12. Blumer D, Benson DF. Personality changes with frontal and temporal lobe lesions. in: Benson DF, Blumer D, eds. Psychiatric Aspects of Neurologic Disease. New York, NY: Grune \& Stratton; 1975:151-169.

13. Damasio AR, Van Hoesen GW. Emotional disturbances associated with focal lesions of the limbic frontal lobe. In: Heilman KM, Satz P, eds. Neuropsychology of Human Emotion. New York, NY: Guilford Press; 1983:85-110.

14. Miller BL, Cummings JL, Villanueva-Meyer J, et al. Frontal lobe degeneration: clinical, neuropsychological, and SPECT characteristics. Neurology. 1991;41: 1374-1382.

15. Cummings JL. Frontal-subcortical circuits and human behavior. Arch Neurol. 1993:50:873-880

16. Mega MS, Cummings JL. Frontal-subcortical circuits and neuropsychiatric disorders. J Neuropsychiatry Clin Neurosci. 1994;6:358-370.

17. McKhann G, Drachman D, Folstein M, et al. Clinical diagnosis of Alzheimer's disease: report of the NINCDS-ADRDA work group under the auspices of Department of Health and Human Services Task Force on Alzheimer's Disease. Neurology. 1984;34:939-944

18. Cummings JL, Mega M, Gray K, Rosenberg-Thompson S, Carusi DA, Gornbein J. The Neuropsychiatric Inventory: comprehensive assessment of psychopathology in dementia. Neurology. 1994;44:2308-2314.

19. Folstein MF, Folstein SE, McHugh PR. 'Mini-mental state': a practical method for grading the cognitive state of patients for the clinician. $J$ Psychiatr Res. 1975;12:189-198.

20. Kanno I, Uemura K, Miura Y. Headtome: a hybrid emission single-photon and positron emission tomograph for imaging of the brain. J Comput Assist Tomogr. 1981:5:216-224

21. Kanno I, Lassen NA. Two methods for calculating regional cerebral blood flow from emission computed tomography of inert gas concentrations. $J$ Comput Assist Tomogr. 1979;5:216-224.

22. Darcourt J, Cauvin JC, Miller BL, Mena I. Absolute rCBF calibration of HMPAO SPECT using Xenon-133. Soc Nucl Med. 1993:18:928.

23. Kuwabara $Y$, Ichiya $Y$, Sasaki $M$, et al. Linealization correction for acetozolamide (Diamox) 99mTc-HMPAO SPECT image: a comparative study with PET. Kaku lgaku. 1994;31:277-282.

24. Miller BL, Itti L, Darby AL, et al. Atrophy-corrected cerebral blood flow in frontotemporal dementia. Ann Gerontol. 1995;9:165-167

25. Damasio H, Damasio AR. Lesion Analysis in Neuropsychology. New York, NY: Oxford University Press Inc; 1989:184-185.

26. Dixon WJ, Brown MB, Engleman L, et al. BMDP Statistical Software. Los Angeles; University of California Press; 1985.

27. Doody RS, Massman P, Mahurin R, Law S. Positive and negative features in Alzheimer's disease. J Neuropsychiatry Clin Neurosci. 1995;7:54-60.

28. Lilly $R$, Cummings $J L$, Benson DF, Frankel $M$. The human Kluver-Bucy syndrome. Neurology. 1983;33:1141-1145.

29. Whitehouse PJ, Price PL, Struble RG, et al. Alzheimer's disease and senile dementia: loss of neurons in the basal forebrain. Science. 1982;215:1237-1239.

30. Forstl H, Burns A, Levy R, Cairns N, Luthert P, Lantos P. Neuropathological correlates of behavioral disturbance in confirmed Alzheimer's disease. $\mathrm{Br} J$ Psychiatry. 1993:163:364-368.

31. Malloy PF, Richardson ED. Assessment of frontal tobe functions. J Neuropsychiatry Clin Neurosci. 1994;6:399-410

32. DeKoskey ST, Scheff SW. Synapse loss in frontal cortex biopsies in Alzheimer's disease: Correlation with cognitive severity. Ann Neurol. 1990;27:457-464.

33. Terry RD, Masliah E, Salmon DP, et al. Physical basis of cognitive alterations in Alzheimer's disease: synapse loss is the major correlate of cognitive impairment. Ann Neurol, 1991;30:572-580.

34. Davies CA, Mann DMA, Sumpter PQ, Yates PO. A quantitative morphometric analysis of the neuronal and synaptic content of the frontal and temporal cortex in patients with Alzheimer's disease. J Neurol Sci. 1987;78:151-164.

35. Masliah E, Terry RD, Alford M, DeTeresa R, Hansen LA. Cortical and subcortical patterns of synaptophysin-like immunoreactivity in Alzheimer disease. Am $J$ Pathol. 1991;138:235-246.

36. Baxter LR, Schwartz JM, Phelps ME, et al. Reduction of prefrontal cortex glucose metabolism common to three types of depression. Arch Gen Psychiatry. 1989:46:243-250.

37. Mayberg HS. Neuro-imaging studies of depression in neurological disease. In Starkstein SE, Robinson RG, eds. Depression in Neurologic Diseases. Baltimore, Md: Johns Hopkins University Press; 1993:186-216.

38. Mayberg HS. Clinical correlates of PET and SPECT identified defects in dementia. J Clin Psychiatry. 1994;55(suppl):12-21. 